\author{
$\$$ Research Square \\ Tatsuki Kaimoto ( $\nabla$ donguriko@hotmail.co.jp ) \\ Tottori Daigaku https://orcid.org/0000-0002-0542-721X \\ Yukihiro Fujita \\ Azabu Daigaku \\ Nobuo Kanno \\ Nihon Jui Seimei Kagaku Daigaku

\section{Brian Beale} \\ Beale Veterinary Specialists and Emergency \\ Yasushi Hara \\ Nihon Jui Seimei Kagaku Daigaku \\ Shinya Yamaguchi \\ YPC tokyo animal orthopeadic Surgical hospital
}

Preprints are preliminary reports that have not undergone peer review.

They should not be considered conclusive, used to inform clinical practice, or referenced by the media as validated information.

\title{
Quantitative evaluation of canine cranial cruciate ligament disease by stress radiography
}

\section{Research article}

Keywords: Canine cranial cruciate ligament disease, Cranial Tibial Displacement Index, Stress radiography

Posted Date: February 25th, 2020

DOI: https://doi.org/10.21203/rs.2.24416/v1

License: (c) (i) This work is licensed under a Creative Commons Attribution 4.0 International License.

Read Full License 


\section{Abstract}

BACKGROUND To establish a quantitative parameter reflecting displacement of the tibia relative to the femoral condyle in dogs with damage to the cranial cruciate ligament ( $\mathrm{CrCL})$.

ANIMALS One hundred and forty-eight client-owned dogs with intact $(n=34)$, partial rupture $(n=43)$, or complete rupture $(n=93)$ of the $\mathrm{CrCL}$ confirmed by arthroscopy of the stifle joint.

PROCEDURES The Cranial Tibial Displacement Index (CTDI) was measured on mediolateral radiographs obtained in lateral recumbency with the tarsal and stifle joints at $90^{\circ}$ of flexion. The sensitivity and specificity of the CTDI for assessment of CrCL damage was compared with that of the cranial drawer test, tibial compression test, fat pad sign, and radiographic OA score.

RESULTS The mean CTDI (mean \pm SD) scores were $0.5 \pm 0.1,0.8 \pm 0.2$, and $1.2 \pm 0.3$ in the groups with an intact, partially ruptured, and completely ruptured $\mathrm{CrCL}$, respectively; the differences between the three study groups were statistically significant $(p<0.001)$. The sensitivity, specificity, and positive and negative predictive values of the CTDI for distinguishing damaged and intact CrCLs were $89.0 \%, 85.3 \%, 96.0 \%$, and $65.9 \%$, respectively.

CONCLUSIONS AND CLINICAL RELEVANCE The CTDI, which quantifies cranial displacement of the tibia, could be an objective indicator reflecting the grade of $\mathrm{CrCL}$ damage. $\mathrm{CrCL}$ injury could be evaluated indirectly from the CTDI score, which may allow a minimally invasive early diagnosis of a ruptured CrCL.

\section{Background}

Rupture of the cranial cruciate ligament ( $\mathrm{CrCL})$ is a common injury in dogs, and the main cause of osteoarthritis $(\mathrm{OA})$ and degenerative disease in the stifle joint ${ }^{1}$. Although rupture of the anterior cruciate ligament can occur acutely with trauma in humans, most cases of $\mathrm{CrCL}$ rupture (CrCLR) occur secondary to chronic degenerative changes combined with chondrometaplasia of the $\mathrm{CrCL}^{2}$. Although the precise mechanism of degeneration of the $\mathrm{CrCL}$ has not been clarified, some causative factors have been reported. These include trauma, immune-mediated mechanisms, age-related degeneration, obesity, an excessive tibial plateau angle (TPA), and conformational abnormalities, such as patellar luxation and a narrowed intercondylar notch ${ }^{3-6}$. These are collectively referred to as $\mathrm{CrCL}$ disease $\mathrm{s}^{2,7}$. However, this disease has been recognized as a degenerative process that ultimately leads to partial or complete ligament rupture, instability, and secondary degenerative joint disease $e^{8,9}$. Instability of the stifle joint following $\mathrm{CrCLR}$ is an important pathophysiologic mechanism leading to development of progressive OA and stifle joint "organ" failure. ${ }^{8,10-13}$ Although an early partial tear is likely to progress to a complete tear if left untreated because of the degenerative cascade of effects that occurs, the benefits of surgery may not outweigh the risks. However, early diagnosis of a $\mathrm{CrCL}$ injury and treatment by tibial-plateau-leveling osteotomy may protect against further disruption of the $\mathrm{CrCL}$, lending stability to the joint and decreasing the risk of meniscal injury and damage to the articular cartilage. ${ }^{14}$ Therefore, for maintenance of normal 
stifle joint function, it is important to diagnose and treat a partial rupture early and prevent transition to osteoarthritis and complete rupture.

A number of techniques and medical imaging systems have been used to diagnose canine CrCLR, including palpation, radiography, arthroscopy, computed tomography (CT), magnetic resonance imaging (MRI), and ultrasonography. In clinical practice, CrCLR is usually diagnosed by orthopedic and radiographic examination. A cranial drawer test (CDT) or tibial compression test (TCT) is used to evaluate craniocaudal instability of the stifle joint ${ }^{15}$. It was determined that the CDT alone or combined with the TCT could not be used to differentiate the cause of stifle instability associated with rupture of the $\mathrm{CrCL}$, caudal cruciate ligament, or total cruciate ligament ${ }^{16}$. It has been pointed out that many dogs with chronic CrCLR do not have palpable instability of the stifle joint because of chronic periarticular fibrosis, and the sensitivity of the CDT and TCT was surprisingly low when performed on conscious patients. ${ }^{17}$ Therefore, more objective tools are needed to accurately detect disorders of the stifle joint. Radiographs showing joint effusion, osteophytosis, or cranial tibial displacement support a diagnosis of CrCLR. Radiologically, there might be evidence of osteophyte formation and/or changes to the infrapatellar fat pad shadow ${ }^{4,18}$. A stress view radiograph of the stifle might be helpful for observing cranial displacement of the tibia in dogs with $\mathrm{CrCLR}^{19}$. Arthroscopy is a diagnostic and therapeutic procedure that provides a direct view of the articular surface for the purpose of integral evaluation of the joint. The disadvantage is that it is an invasive procedure that requires major technologic infrastructure and many hours of specialized training ${ }^{20}$. Ultrasonography, which involves non-invasive, non-ionizing radiation and is portable and less expensive, is a useful technique for evaluation of intraarticular proliferation of reactive fibrotic tissue in unstable stifle joints with CrCLR as a result of chronic synovitis ${ }^{21}$. However, expert operators are mandatory for ultrasonography to be useful. Furthermore, ultrasonography is not an accurate test for CrCLR, but is specific for the pathologic changes in the soft tissue that occur as a consequence of joint instability ${ }^{21,22}$. Other useful noninvasive techniques are scintigraphy ${ }^{23}, \mathrm{CT}$, and $\mathrm{MRI}^{24}$; however, these modalities are not routinely available in veterinary medicine. Arthroscopy and MRI may be useful for confirming the diagnosis; however, arthroscopy is invasive and MRI is expensive ${ }^{23,25}$. Meanwhile, CT emits a high level of radiation and has limited ability to detect a fracture. Again, CT and MRI require expert operators to be useful. Undeniably, all these medical imaging systems have some disadvantages.

We have developed a quantitative method to evaluate instability of the stifle joint related to insufficiency of the CrCL. We evaluated and compared the extent of $\mathrm{CrCL}$ damage using an index measured on radiographs taken in the stress position in dogs with intact stifle joints and in those with partial or complete CrCLR confirmed by arthroscopy. The objective of this study was to evaluate the efficacy of this new technique for diagnosis of CrCLR

\section{Materials And Methods}

Clinical cases 
All subjects in the study were client-owned dogs that were presented at our clinic (YPC Tokyo Animal Orthopedic Surgical Hospital, Koutoku, Tokyo) between January 2015 and May 2017. One hundred and twenty six dogs have hind limb lameness and were diagnosed to have $\mathrm{CrCL}$ disease while 22 dogs were selected as a control group with no lameness of four limbs or opposite side of CrCLR's limb and were not diagnosed to have $\mathrm{CrCL}$ disease by arthroscopy. Complete medical records were available for all dogs. The inclusion criteria were canine species, diagnosed or not diagnosed to $\mathrm{CrCLR}^{4-6}$, mediolateral radiographs of the stifle joint, including the tibia, obtained while not under anesthesia, and results of clinical examination available. Information on duration of lameness of the affected limb was obtained from the owner. All the owners provided informed consent, and participation in the study did not impact decisions regarding clinical management.

\section{Study groups}

The study population included a control group, a group with partial CrCLR, and a group with complete CrCLR. The control group consisted of 10 dogs which have CrCLR on the opposite side and 12 no abnomal dogs without any orthopedic disease that were presented to our hospital. The 34 hind limbs in this group were confirmed to be healthy on the basis of a normal physical examination (with no lameness) and normal orthopedic, radiographic, and arthroscopic examination results. Our hospital is secondary hospital and specializes in orthopedic surgery especially stifle arthroscopy, so some aggressive owners come to our hospital to inspect their dogs with stifle arthroscopy and we always performed arthroscopy to both stifles. In other words, we performed arthroscopy to 10 dogs (10 hind limbs) as check of the opposite side of hind limb sufferd from CrCLR and to 12 dogs (24 hind limbs) under extremely strong client's desire. Dogs in the CrCLR group had stifle joints with naturally occurring damage to the $\mathrm{CrCL}$ confirmed by arthroscopy and no evidence of other stifle pathology on physical or radiographic examination. The diagnosis of CrCLR was made arthroscopically and confirmed intraoperatively.

\section{Arthroscopy}

All arthroscopy procedures (170 stifles in 148 dogs) were performed under general anesthesia by the same surgeon (SY) in accordance with Whitney ${ }^{26}$ and Beale et al. ${ }^{20}$ using 1.9-mm or 2.4-mm Karl Storz ${ }^{\circledR}$ arthroscopes with 30 degrees of angulation (Karl Storz GmbH \& Co. KG, Tuttlingen, Germany) for synovitis or disruption of the cranial or caudal cruciate ligament, articular surface of the trochlear ridge, or meniscus ${ }^{27-29}$. The $\mathrm{CrCL}$ was graded as intact (no damage, with continuity), partially ruptured (damage present, continuity preserved), or completely ruptured (damage present, continuity disrupted). The majority of the lesions were close to the femoral insertion site and there were no distal lesions (Fig. 1). At last, all inspection including arthroscopy was conducted under orner's consent. In particularly, the dogs classified as normal were inspected by orner's strong request.

Stress radiography 
Radiography was performed without sedation or anesthesia. During this examination, we referred to the TCT, which is an orthopedic procedure performed at the time of clinical diagnosis of CrCLR. Mediolateral radiographs were obtained with the dogs in lateral recumbency and the tarsal and stifle joints at $90^{\circ}$ of flexion with the tibia parallel to the digital image capture device. The X-ray beam was centered over the stifle joint and collimated to the tarsus, entire tibia, and distal half of the femur. If the intercondylar eminences were not exactly superimposed, the midpoint of each eminence was identified. The femoral condyles and talar trochlea were superimposed to achieve the correct rotational alignment. The radiographs were obtained using a digital system and assessed using a DICOM viewer (Sedecal, Entrad). Radiographs of the stifle joints with superimposition of the femoral condyles $\leq 2 \mathrm{~mm}$ and flexion angles of $60^{\circ}-120^{\circ}$ were assessed. ${ }^{30}$ If the femoral condyles were not exactly superimposed, a best-fit circle was drawn around each femoral condyle and the center of each condyle was identified.

The radiographs were digitized for analysis and analyzed using the DICOM viewer software. The cranial and caudal aspects of the medial tibial condyle were used as landmarks for the proximal tibial joint orientation line (Fig. 2) $)^{31,32}$ and the distal aspect of the intermediate ridge of the tibia cranially and caudodistal aspect of the cochlea tibia were used as landmarks for the distal tibial joint orientation line (Fig. 3). The landmarks of each joint surface were connected by a line to create the proximal and distal joint orientation lines. The anatomic and mechanical axes of the tibia were identified ${ }^{33-35}$. The mechanical axis defined as the straight line extending from the midpoint between the apices of the two tibial intercondylar eminences of the tibial plateau to the center of the circle created by the tarsus was used, as described elsewhere $34,36,37$. The tibial anatomic axis was defined as the line connecting the midpoint between the cranial and caudal cortex of the tibia at $50 \%$ and $75 \%$ of the length of the tibial shaft (Fig. 4). ${ }^{38-41}$ All measurements were made by the same investigator (TK).

The angle of the stifle joint was measured as the angle formed between the long axis of the distal femur and the tibia in all cases ${ }^{42}$. This angle ("O") was determined by drawing a segment $(\mathrm{FW})$ between the femoral cortices at a distance equal to the length of the femoral condyle from the proximal extent of the trochlea. A segment ("D") parallel to the FW was drawn $20 \mathrm{~mm}$ proximal to the FW. A line was then drawn joining the centers of the FW and D segments to define the long axis of the distal femur. The anatomic axis of the tibia was drawn as previously defined. The stifle joint angle was measured at the intersection of these two axes (Fig. 5). In all stifles, the actual angle of the tarsal joint were also measured from the two long axes of the previous tibial axis and the tarsal axis that was defined as the lines between the two shafts, midpoints at the intertarsal joint, and the tarsal over metacarpal joint (Fig. 6) ${ }^{30}$. For all stifle joints, the TPA was measured on a mediolateral radiograph acquired in the stress position according to the method devised by Warzee et $\mathrm{al}^{43}$.

Measurement of Cranial Tibial Displacement Index

A circle was drawn on the mediolateral radiograph to fit the circumference of the femoral condyles (the average of the circle both lateral and medial femoral condyles, more fit in femoral condyles). The center of the femoral condyle was then determined. Next, the radius of the femoral condyle (C) was measured in 
centimeters. A line was drawn from the center of the femoral condyle parallel to the line of the mechanical axis of the tibia. The distance ( $a$, in centimeters) between them was then measured. The ratio of a to $\mathrm{C}$, i.e., the tibial cranial displacement index (CTDI), was calculated to correct for differences in body size (Fig. 7).

We determined the cut-off CTDI values for intact and partially ruptured CrCLs. In the present study, to determine the normal range of the CTDI, we initially excluded measurements obtained at the extremes on either side and then calculated the mean of the values obtained in the normal group.

Palpation

The CDT and TCT were performed in lateral recumbency without sedation or anesthesia in all cases using the techniques and procedures described, respectively, by Jerram and Walker ${ }^{44}$ and Henderson and Milton ${ }^{45}$. These tests were repeated with the stifle held in varying degrees of extension and flexion. ${ }^{45}$ The results of the tests were represented as positive or negative.

Fat pad sign

Synovial effusion and osteophytosis were used to evaluate the severity of OA of the stifle joint. The fat pad sign was evaluated on mediolateral radiographs and defined as soft tissue opacity in the cranial part of the stifle joint extending cranially to a line drawn perpendicularly from the cranial margin of the medial tibial condyle. This line estimates the cranial margin of the cranial poles of the menisci. Cranial extension of soft tissue opacity beyond this location was deemed excessive. Synovial effusion was graded subjectively on a 0 -2-point scale (0, normal; 1 , mild; 2 , severe $)^{46,47}$.

Radiographic OA score

The severity of OA in the stifle joints of dogs in the CrCLR group was graded on radiographs using an established scoring system ${ }^{48}$. Scores obtained using this system range from 0 (no OA sign, i.e., osteophytes absent) to 4 (severe, osteophytes present on the patella, femoral trochlear groove, medial and lateral femoral condyles, and tibial plateau, i.e., subchondral sclerosis of the femoral condyles).

Sensitivity, specificity, and positive and negative predictive values

The sensitivity, specificity, positive predictive value (PPV), and negative predictive value (NPV) of the CDT, TCT, fat pad sign, ROS, and CTDI in the diagnosis of CrCL were compared. For the CDT and TCT, the result was recorded as "positive" (over 0 ) or "negative". CTDI cut-off values of 0.62 and 1.01 were identified as the thresholds for an intact CrCL and a partial CrCLR, respectively. Sensitivity is defined as the proportion of true-positive results identified correctly and specificity is the proportion of true-negative results identified correctly. The PPV is the proportion of positive tests that are truly positive and the NPV is the proportion of negative tests that are truly negative. 
Tukey's honestly significant difference (HSD) test and the Games-Howell test were used to identify statistically significant between-group differences in patient age and body weight, joint angle, and TPA between the three study groups. The relationships between arthroscopic $\mathrm{CrCL}$ grade and the study variables ( $a, C, a / C$ ratio, stifle angle, tarsal angle, TPA, age, body weight, and duration of lameness) were determined using the Spearman's rank correlation. The data are presented as the mean and standard deviation or as the number or percentage. A p-value $<0.05$ was considered statistically significant for all comparisons.

\section{Results}

Arthroscopy

Thirty-four stifle joints were classified as intact (no damage or vascularization of the $\mathrm{CrCL}$, complete continuity), 43 as partially ruptured (various degrees of damage and vascularization, most being close to the femoral insertion site, but preserved continuity of the $\mathrm{CrCL}$ ), and 93 as completely ruptured. The caudal cruciate ligament was confirmed to be intact by arthroscopic observation in all three-study groups (Table 1).

Demographic and clinical data

The 148 dogs were divided into an intact group, partial rupture group, and complete rupture group based on arthroscopic and intraoperative findings. Detailed information on sex, age, and body weight are provided in Table 1.

The intact group consisted of 22 dogs (34 stifles) of mean age $6.0 \pm 3.1$ (range, $0.92-13.7$ ) years and included 3 sexually intact females, 8 neutered females, 3 sexually intact males, and 8 neutered males. The mean body weight was $9.2 \pm 7.4$ (range, 2.4-30.0) $\mathrm{kg}$. There were 11 breeds including American Cocker Spaniel $(n=1)$, American pit bull $(n=2)$, Border collie $(n=2)$, Chihuahua $(n=6)$, Golden Retriever $(n=2)$, Labrador retriever $(n=1)$, mixed breeds $(n=4)$, Pembroke Welsh Corgi $(n=2)$, Pyrenean Sheepdog $(n=2)$, Shiba $(n=5)$, and Toy poodle $(n=7)$. The stifle joint's breakdown was that the right sides were 17 cases and the left sides were 17 cases.

The partial rupture group consisted of 39 dogs (43 stifles) of mean age $6.6 \pm 3.8$ (range, 2.0-14.2) years, including two sexually intact females, 19 neutered females, 2 sexually intact males, and 16 neutered males. The mean body weight was $19.9 \pm 15.5$ (range, 2.3-65.0) $\mathrm{kg}$. There were 21 breeds represented, including American Cocker Spaniel $(n=2)$, Akita $(n=1)$, American Pit Bull $(n=3)$, Australian cattle dog $(n$ $=1)$, Beagle $(n=2)$, Bernese Mountain Dog $(n=2)$, Bichon Frise $(n=1)$, Chihuahua $(n=2)$, Doberman $(n=$ 1), Golden Retriever $(n=1)$, Great Dane $(n=1)$, Labrador Retriever $(n=3)$, mixed breeds $(n=3)$, Newfoundland $(n=1)$, Old English Sheepdog $(n=1)$, Pembroke Welsh Corgi $(n=4)$, Rough Collie $(n=1)$, Siberian Husky $(n=1)$, Toy poodle $(n=6)$, and Yorkshire Terrier $(n=2)$. The stifle joint lesions were on the 
right in 17 cases and on the left in 26 . The mean duration of lameness was $48.1 \pm 68.4$ (range, 1.0-240) days.

The complete rupture group consisted of 87 dogs (93 stifles) of mean age $7.7 \pm 3.1$ (range, 1.0 -14.0 ) years, including 12 sexually intact females, 42 neutered females, 9 sexually intact males, and 24 neutered males. The mean body weight was $16.3 \pm 13.2$ (range, $2.0-70.0$ ) $\mathrm{kg}$. There were 20 breeds represented, including Akita $(n=1)$, American Cocker Spaniel $(n=7)$, Beagle $(n=1)$, Border Collie $(n=6)$, Bernese Mountain Dog $(n=3)$, Cavalier King Charles Spaniel $(n=1)$, Chihuahua $(n=10)$, Dobermann $(n=1)$, Golden Retriever $(n=6)$, Husky $(n=1)$, Jack Russell Terrier $(n=4)$, Labrador Retriever $(n=8)$, Mixed breeds $(n=13)$, Papillon $(n=1)$, Pembroke Welsh Corgi $(n=7)$, Pomeranian $(n=1)$, Shiba $(n=1)$, Toy Poodle $(n=$ 9), Welsh Terrier $(n=1)$ and Yorkshire Terrier $(n=5)$. The stifle joint lesions were on the right in 43 cases and on the left in 50. The mean duration of lameness was $26.7 \pm 37.3$ (range, $0-200$ ) days.

There was no significant between-group difference in age (intact vs complete, $\mathrm{P}=0.029$; intact vs partial, $P=0.682$; partial vs complete, $P=0.184$ ). The dogs in the complete and partial rupture groups were significantly heavier than those in the intact group (both $P=0.001$ ).

Tibial plateau angle

The mean TPA was $25.4 \pm 3.9^{\circ}$ (range, $14.6^{\circ}-32.2^{\circ}$ ) in the intact group, $27.0^{\circ} \pm 4.3^{\circ}$ (range, $13.5^{\circ}-37.0^{\circ}$ ) in the partial rupture group, and $28.6^{\circ} \pm 4.2^{\circ}$ (range, $20.0^{\circ}-37.0^{\circ}$ ) in the complete rupture group (Table 1 ). There was a significant difference in TPA between the intact group and the complete rupture group $(\mathrm{P}=$ $0.001)$ but not between the intact and partial rupture groups or between the partial and complete rupture groups $(P=0.21$ and $P=0.1$, respectively $)$.

Cranial Tibial Displacement Index

In the intact group, the mean a value was $3.0 \pm 1.1 \mathrm{~mm}$, the mean $C$ value was $6.0 \pm 0.3 \mathrm{~mm}$, and the mean CTDI score was $0.5 \pm 0.1$; the respective values were $5.8 \pm 2.2 \mathrm{~mm}, 7.9 \pm 0.4 \mathrm{~mm}$, and $0.8 \pm 0.2$ in the partial rupture group and $8.3 \pm 3.0 \mathrm{~mm}, 7.3 \pm 0.2 \mathrm{~mm}$, and $1.2 \pm 0.3$ in the complete rupture group. The mean CTDI cut-off values for distinguishing between the partial and complete rupture groups and between the intact and partial rupture groups were 1.01 and 0.62 , respectively. The radius of the femoral condyle was significantly larger in the complete and partial rupture groups than in the intact group $(P=$ 0.002 and $P=0.011$, respectively), with no significant difference between the partial rupture group and the complete rupture group $(P=0.38)$. There were significant differences in the a value and CTDI score between the three study groups $(P<0.001$; Table 2$)$

The mean actual stifle angle on stress radiography was $88.4^{\circ} \pm 10.5^{\circ}$ (range, $67.0^{\circ}-120^{\circ}$ ) in the intact group, $83.1^{\circ} \pm 9.0^{\circ}$ (range, $64.4^{\circ}-106.1^{\circ}$ ) in the partial rupture group, and $86.4^{\circ} \pm 10.4^{\circ}$ (range, $64.0^{\circ}-$ $117.2^{\circ}$ ) in the complete rupture group. The mean actual tarsal angle was $93.5^{\circ} \pm 6.8^{\circ}$ (range, $81.4^{\circ}-$ $110.2^{\circ}$ ) in the intact group, $92.2^{\circ} \pm 7.0^{\circ}$ (range, $79.5^{\circ}-109.8^{\circ}$ ) in the partial rupture group, and $89.7^{\circ} \pm 7.7^{\circ}$ (range, $68.1^{\circ}-116.1^{\circ}$ ) in the complete rupture group (Table 1). 
There were no significant between-group differences in the stifle angle (intact vs complete, $\mathrm{P}=0.458$; intact vs partial, $P=0.05$; partial vs complete, $P=0.232$ ). The tarsal joint angle was significantly smaller in the complete rupture group than in the intact group $(P=0.002)$; however, there was no significant difference between the intact and partial rupture groups $(P=0.433)$ or between the partial and complete rupture groups $(P=0.076)$.

\section{Palpation}

The CDT and TCT results were negative in all dogs in the intact group. In the partial rupture group, the CDT result was positive in $67.6 \%$ of dogs, the mean CTDI scores in the joints that were CDT-positive and CDT-negative were $0.8 \pm 0.3$ and $0.8 \pm 0.2$, respectively, the TCT result was positive in $52.9 \%$ of cases, and the mean CTDI scores in the joints that were TCT-positive and TCT-negative were $0.8 \pm 0.3$ and $0.8 \pm 0.2$, respectively (Fig. 9). In the complete rupture group, the CDT result was positive in $94.4 \%$ of dogs, the mean CTDI scores in the joints that were CDT-positive and CDT-negative were $1.2 \pm 0.3$ and $0.8 \pm 0.2$, respectively, the TCT results was positive in $93.1 \%$ of cases, and the mean CTDI scores in the joints that were TCT-positive and TCT-negative were $1.2 \pm 0.3$ and $0.9 \pm 0.2$, respectively.

Correlations between CTDI and the variables measured were determined, and the results are summarized in Table 3. No association of CTDI with age, sex, angle, TPA, weight, or duration of lameness was detected in any of the study groups (Table 2).

Fat pad sign

None of the dogs in the intact group had the fat pad sign. In the partial rupture group, a grade 0 fat pad sign was present in $25.6 \%$ of cases, a grade 1 sign in $39.5 \%$, and a grade 2 sign in $34.9 \%$. The mean CTDI score was $0.8 \pm 0.3$ for the joints with a grade 0 fat pad sign, $0.7 \pm 0.2$ for those with a grade 1 sign, and $0.8 \pm 0.2$ for those with a grade 2 sign. In the complete rupture group, a grade 0 fat pad sign was present in $16.1 \%$ of cases, a grade 1 sign in $41.9 \%$, and a grade 2 sign in $41.9 \%$. The mean CTDI score was $1.2 \pm$ 0.3 for the joints with a grade 0 fat pad sign, $1.2 \pm 0.4$ for those with a grade 1 sign, and $1.2 \pm 0.3$ for those with a grade 2 sign.

ROS

ROS were not detected in any of the cases in the intact group. In the complete rupture group, the mean ROS was 2.5 (range, $0-4$ ). Twelve joints were graded as ROS (0), 12 as (1), 18 as (2), 17 as (3), and 34 as (4) is 34. The mean CTDI score for the joints graded as ROS (0) was $1.2 \pm 0.3$, that for ROS (1) was $1.2 \pm$ 0.4 , that for ROS (2) was $1.2 \pm 0.4$, that for ROS (3) was $1.2 \pm 0.3$, and that for ROS (4) was $1.1 \pm 0.3$. In the partial rupture group, the mean ROS was 0.74 (range, $0-3$ ). Nineteen joints were graded as ROS (0), 17 as ROS (1), six as ROS (2), one as ROS (3), and none as ROS (4). The mean CTDI score for the joints graded as ROS (0) was $0.7 \pm 0.2$, that for ROS ( 1 ) was $0.8 \pm 0.2$, that for ROS (2) was $0.7 \pm 0.2$, that for ROS (3) was 0.8 (Tables 2 and 3 ). The ROS scores were significantly higher in the complete rupture group than in 
the partial rupture group. There were no significant differences in these variables between CTDI in each ROS in this study.

Sensitivity, specificity, PPV, and NPV

The sensitivity, specificity, PPVs and NPVs values for the CDT, TCT, fat pad sign, ROS, and CTDI score are summarized in Table 4.

\section{Discussion}

There was a significant difference in the CTDI score between the intact group, partial rupture group, and complete rupture group in this study, suggesting that the CTDI, which quantifies the cranial displacement of the tibia relative to the femoral condyle, could be an objective indicator of the degree of CrCL damage. Our present findings suggest that we may be able to indirectly evaluate damage to the $\mathrm{CrCL}$ from the CTDI score, potentially enabling minimally invasive early diagnosis of CrCLR (Fig. 10).

The significant difference in the femoral radius between the partial rupture group and the intact group may reflect differences in body weight and physique. The tarsal joint angle was significantly lower in the complete rupture group than in intact group. The target joint angle on radiography was set to $90^{\circ}$. We adopted this stored position taking into account the reproducibility at the time of acquiring the images. However, in CrCLR cases, the tibia is displaced forward and the anatomic axis is also tilted forward, so the tarsal joint tends to flex easily ${ }^{24}$. Although the influence of the difference in the tarsal joint angle is unknown, it is considered that the $90^{\circ}$ imaging method by visual observation is useful because a significant difference in the CTDI score was found in this study. However, in a recent report, it was shown that cranial displacement of the tibia is maximized when the tarsal joint is overflexed. ${ }^{24}$ Measurement at the time of overflexion should be included in a further study, because it is possible that the difference in CTDI due to the degree of damage will become more awkward.

It is considered that various factors, including TPA, OA scores, the hamstring muscles, medial buttress, periarticular fibrosis, and intra-articular structure, would have an influence on the $\mathrm{CTDI}^{49-54}$. There are several studies to demonstrate that these factors affect CrTT and lead to degenerative joint disease and CrCLR ${ }^{55-62}$. Especially as for muscle tension, our study implements under no anesthesia. In this circumstance, some dogs are not corporate inspection because of such as fear or tension, may not indicate true CTDI. It is considered to be an issue. When the CrCLR is chronic and the circumference of the joint has become fibrotic and fluctuation is reduced, $90^{\circ}$ of stress during palpation or radiography may reduce the thrust forward propulsion force. No correlation was found between the days since onset and the CTDI score in our study. However, the CTDI score may be decreased in cases where the stifle joint is stabilized by periarticular fibrosis because of a chronic course. Furthermore, in our study, the complete rupture group had a higher TPA than the intact group. CrTT is generated by loading the body weight to the stifle joint surface and TPA. Increases in posterior inclination of the tibial plateau have been associated with increases in cranial translation of the tibia during monopodial stance in humans ${ }^{63}$. This finding 
suggests a similar situation in canine stifle joints. When a stifle joint in a dog with CrCLR show greater posterior inclination of the tibial plateau, cranial translation of the tibia might increase. In previous experiments using canine cadavers, the tibial plateau leveling procedure was consistently successful in eliminating cranial translation of the tibia during axial loading ${ }^{43,55}$. This finding means that the CrTT could change with the degree of TPA, and a greater CrTT means greater CTDI. However, it is difficult to quantify the force generated by the hamstring muscles, the antagonistic effects of the medial buttress, and periarticular fibrosis against CrTT.

The fat pad sign, CDT, and TCT are diagnostic tests for CrCLR in clinical practice, and have been extensively studied ${ }^{65}$. Based on that earlier study, we compared the positive rates for each examination performed in this study. In our study, retention of joint fluid was confirmed at about $80 \%$ of joints in the complete rupture group, $93 \%$ of which showed no abnormality in CDT or TCT. However, in the partial rupture group, about $30 \%$ did not show retention of joint fluid and no abnormality was detected in palpation. Therefore, we compared the CTDI with the fat pad sign with or without palpation in the complete rupture group and the partial rupture group. In the complete rupture group, only four cases that were negative by palpation were mildly low, but the other values were similar in the other $95 \%$ of cases. In the partial rupture group, the CTDI score was almost the same regardless of the level of joint fluid retention or findings on palpation. Therefore, it is possible that the CTDI can evaluate damage that cannot be detected by palpation or the fat pad sign. According to a study of humans, the partial rupture is categorized as $25-50 \%$ or $50-75 \%$ ligament rupture ${ }^{64}$. On the other hand, in veterinary orthopedics, partial rupture of the canine $\mathrm{CrCL}$ is classified clinically as functioning or non-functioning depending on the function of the remaining ligament as qualitative. So study of the relationship between the CTDI score and the degree of damage as quantitative is needed in the future.

The sensitivity of the CTDI was higher than that of the CDT, TCT, fat pad sign, and ROS. We evaluated the fat pad sign as an indicator of OA of the stifle joint in the present study. The criteria used were selected based on high reproducibility ${ }^{37,38}$. The fat pad sign is one of the earliest and most consistent findings in dogs with CrCLR and is consistent with joint effusion, edema of the fat pad, or periarticular fibrosis ${ }^{50-52}$. However, the fat pad sign and ROS only evaluate evidence of secondary arthritis of the stifle joint such as tumor or IMPA, so are not specific indicators of CrCLR.

Moreover, both the CDT and TCT have the potential to yield false-negative results. I suggested that both these tests gave similar results for a recent $\operatorname{CrCLR}$, but are unreliable in the diagnosis of chronic failure of the $\mathrm{CrCL}^{4,44,66}$. There is no single test or sign has been shown to be universally applicable or reliable. In summary, measurement of the CTDI may yield more objective results and a more reliable diagnosis.

In one study, the sensitivity of the CDT when performed on conscious patients was surprisingly low. The CDT in conscious dogs is perhaps the most commonly used diagnostic test for CrCLR, and the findings of our study indicate that reliance on this test in conscious patients will result in significant numbers of $\mathrm{CrCL}$ cases being misdiagnosed as cruciate-normal, and $40 \%$ of dogs with proven $\mathrm{CrCL}$ failure do not have cranial instability detectable by the $\mathrm{CDT} .{ }^{17}$ It is normal that craniocaudal movement beyond the $0-$ 
$2 \mathrm{~mm}$ of mobility found in a normal stifle joint. On the other hand, if a partial tear is present, the cranial drawer sign may reveal only $2-3 \mathrm{~mm}$ of instability when the test is performed with the stifle flexed and no instability with the stifle in extension ${ }^{5}$. In addition, one study found that 12 of 25 dogs with partial rupture of the $\mathrm{CrCL}$ had no detectable cranial drawer sign in response to manipulation of the involved stifle ${ }^{67}$. Hence, it is not surprising that veterinarians encounter difficulties in diagnosing partial ruptures of the $\mathrm{CrCL}$ with palpation. Subjective evaluation on palpation frequently varied between observers and agreed poorly. Therefore, a diagnosis of CrCLR may be qualitative and subjective. Our results show that CTDI may be an objective index for diagnosis of CrCLR under no anesthesia and sedation with minimally invasive.

\section{Conclusion}

In our study, radiograph showed that the CTDI may be the most beneficial, objective, and minimally invasive diagnostic technique for indirect evaluation of the CrCL.

However, our study has some limitations and was performed in a small population, so further research is needed to identify differences according to canine breed, sex, age, muscle strength in the hind leg as well as the influence of joint fibrosis, comparison of the diagnosis rate using the fat pad sign or palpation, and classification of a partial tear.

\section{Abbreviations}

CDT cranial drawer test

$\mathrm{CrCL} \quad$ cranial cruciate ligament

CrCLR cranial cruciate ligament rupture

CrTT cranial tibial thrust

CT computed tomography

CTDI Cranial Tibial Displacement Index

MRI magnetic resonance imaging

OA osteoarthritis

ROS radiographic OA score

TCT tibial compression test

TPA tibial plateau angle 


\section{Declarations}

\section{Ethics approval and consent to participate}

This study was conducted in accordance with the Declaration of Basel.

Written informed consent was obtained from all the study participants, including consent to participate and to publish the findings.

\section{Consent for publication}

Not applicable

\section{Availability of data and materials}

The datasets used and/or analyzed during the current study are available from the corresponding author on reasonable request.

\section{Competing interests}

The authors declare that they have no competing interests.

\section{Funding}

No funding.

I correct and analysis the data by myself.

\section{Authors' contributions}

T.K.: Conception of study, Study design, Acquisition of data

Data analysis \& interpretation: T.K., N.K.

Drafting or revising of manuscript: Y.F., N.K.

Approval of submitted manuscript: Y.F., N.K., B.B., Y.H., S.Y.

Publicly accountable for relevant content: F.Y., B.B., Y.H., S.Y.

\section{Acknowledgments}

No third party funding or support was received for this study or for the writing or publication of the manuscript. The authors declare that there were no conflicts of interest. The authors thank Editage (www.editage.jp) for English language editing.

\section{Authors' information (optional)}


I am a clinical veterinarian for five years in Japan.Now I work at an animal orthopedic hospital as a veterinarian after I worked at Tokyo University as a resident.

During the student at Tottori University, I worked on TRPA1 channel under the guidance of Pharmacology professor Ohta T.

Last year I presented a poster at ACVS in Pheonix and oral presentation at AiSVS.

Now I have submitted the paper about CrCLR to BMC with Dr. Brian Beale who is a small animal surgical specialist in the US.

\section{Footnote}

OsiriX DICOM Viewer (Pixmeo SARL, Geneva, Switzerland).

\section{References}

1. Vasseur PB, Berry CR. Progression of stifle osteoarthrosis following reconstruction of the cranial cruciate ligament in 21 dogs. J Am Anim Hosp Assoc 1992;28:129-136.

2. Duval JM, Budsberg SC, Flo GL, et al. Breed, sex, and body weight as risk factors for rupture of the cranial cruciate ligament in young dogs. J Am Vet Med Assoc 1999;215:811-814.

3. Lampman TJ, Lund EM, Lipowitz AJ. Cranial cruciate disease: current status of diagnosis, surgery, and risk for disease. Vet Comp Orthop Traumatol 2003;16:122-126.

4. Moore KW, Read RA. Rupture of the cranial cruciate ligament in dogs. Part II. Diagnosis and management. Compend Contin Educ Pract Vet 1996;18:381-391.

5. Vasseur P. The stifle joint. In: Textbook of Small Animal Surgery. Slatter D, editor. 3rd ed. Philadelphia: Saunders; 2003.

6. Comeford EJ, Tarlton JF, Avery NC, et al. Distal femoral intercondylar notch dimensions and their relationship to composition and metabolism of the canine anterior cruciate ligament. Osteoarthritis Cartilage 2006;14:273-278.

7. Bennett $D$, Tennant B, Lewis DG, et al. A reappraisal of anterior cruciate ligament disease in the dog. J Small Anim Pract 1988;29:275-297.

8. Comerford EJ, Smith K, Hayashi K. Update on the aetiopathogenesis of canine cranial cruciate ligament disease. Vet Comp Orthop Traumatol 2011;24:91-98.

9. Hayashi K, Manley P, Muir P. Cranial cruciate ligament pathophysiology in dogs with cruciate disease: a review. J Am Anim Hosp Assoc 2004;40:385-390.

10. Slocum B, Devine T. Cranial tibial thrust: a primary force in the canine stifle. J Am Vet Med Assoc 1983;183:456-459.

11. Kim SE, Pozzi A, Kowaleski MP, et al. Tibial osteotomies for cranial cruciate ligament insufficiency in dogs. Vet Surg 2008;37:111-125. 
12. Cook JL. Cranial cruciate ligament disease in dogs: Biology versus biomechanics. Vet Surg 2010;39:270-277.

13. Griffon DJ. A review of the pathogenesis of canine cranial cruciate ligament disease as a basis for future preventive strategies. Vet Surg 2010;39:399-409.

14. Hulse D, Beale B, Kerwin S. Second look arthroscopic findings after tibial plateau leveling osteotomy. Vet Surg 2010;39:350-354.

15. Might KR, Bachelez A, Martinez SA, et al. Evaluation of the drawer test and the tibial compression test for differentiating between cranial and caudal stifle subluxation associated with cruciate ligament instability. Vet Surg 2013;42:392-397.

16. Pond MJ, Campbell JR. The canine stifle joint I. Rupture of the anterior cruciate ligament: an assessment of conservative and surgical treatment. J Small Anim Pract 1972;13:1-10.

17. Carobbi B, Ness MG. Preliminary study evaluating tests used to diagnose canine cranial cruciate ligament failure. J Small Anim Pract 2009;50:224-226.

18. Jerram RM, Walker AM. Cranial cruciate ligament injury in the dog: pathophysiology, diagnosis and treatment. NZ Vet J 2003;51:149-158.

19. de Rooster $H$, Van Ryssen $B$, van Bree H. Diagnosis of cranial cruciate ligament injury in dogs by tibial compression radiography. Vet Rec 1998;142:366-368.

20. Beale BS, Hulse DA, Schulz KS, et al. Small Animal Arthroscopy (2003). doi:10.1016/B978-0-72168969-2.X5001-9

21. Gnudi G, Bertoni G. Echographic examination of the stifle joint affected by cranial cruciate ligament rupture in the dog. Vet Radiol Ultrasound 2001;42:266-270.

22. Kramer M, Stengel H, Gerwing M, et al. Sonography of the canine stifle. Vet Radiol Ultrasound 1999;40:282-293.

23. Baird DK. Low-field magnetic resonance imaging of the canine stifle joint: normal anatomy. Vet Radiol Ultrasound 1998;39:87-97.

24. Pujol E, Van Bree $H$, Cauzinille $L$, et al. Anatomic study of the canine stifle using low-field magnetic resonance imaging (MRI) and MRI arthrography. Vet Surg 2011;40:395-401.

25. Banfield CM, Morrison WB. Magnetic resonance arthrography of the canine stifle joint: Technique and applications in eleven military dogs. Vet Radiol Ultrasound 2000;41:200-213.

26. Whitney, WO. Arthroscopic reconstruction of the cranial cruciate ligament in the dog. In: Proceedings of the Eighth Annual ACVS Symposium, Chicago, IL, USA, 1998.

27. Cook CR. Ultrasound imaging of the musculoskeletal system. Vet Clin North Am Small Anim Pract 2016;46:355-371.

28. Little JP, Bleedorn JA, Sutherland BJ, et al. Arthroscopic assessment of stifle synovitis in dogs with cranial cruciate ligament rupture. PLoS One 9, (2014).

29. Nečas A, Srnec R, Kecová H. Diagnostic reliability of stifle arthroscopy of pathological changes in cruciate deficient knee. Acta Vet Brno 2002;71:249-254. 
30. Lee $\mathrm{JH}$, Jeong SW. Feasibility of utilizing the patellar ligament angle for assessing cranial cruciate ligament rupture in dogs. $J$ Vet Sci 2014;15:563-568.

31. Reif $U$, Probst CW. Comparison of tibial plateau angles in normal and cranial cruciate deficient stifles of Labrador retrievers. Vet Surg 2003;32:385-389.

32. Slocum B, Slocum TD. Tibial plateau leveling osteotomy for repair of cranial cruciate ligament rupture in the canine. Vet Clin North Am Small Anim Pract 1993;23:777-795.

33. Han HS, Chang CB, Seong SC, et al. Evaluation of anatomic references for tibial sagittal alignment in total knee arthroplasty. Knee Surg Sports Traumatol Arthrosc 2008;16:373-377.

34. Paley D. Principles of deformity correction: what is alignment and malaligment. Improv Accuracy Knee Arthroplast. 2-4 (2012).

35. Shao JJ, Parker Vail T, Wang QJ, et al. Anatomical references for tibial sagittal alignment in total knee arthroplasty: a comparison of three anatomical axes based on 3D reconstructed CT images. Chin Med J (Engl) 2013;126:3840-3844.

36. Baroni E, Matthias RR, Marcellin-Little DJ, et al. Comparison of radiographic assessments of the tibial plateau slope in dogs. Am J Vet Res. 2003;64:586-589.

37. Bailey CJ, Smith BA, Black AP. Geometric implications of the tibial wedge osteotomy for the treatment of cranial cruciate ligament disease in dogs. Vet Comp Orthop Traumato/2007;20:169174.

38. Osmond CS, Marcellin-Little DJ, Harrysson OL, et al. Morphometric assessment of the proximal portion of the tibia in dogs with and without cranial cruciate ligament rupture. Vet Radiol Ultrasound 2006;47:136-141.

39. Dismukes DI, Tomlinson JL, Fox DB, et al. Radiographic measurement of canine tibial angles in the sagittal plane. Vet Surg 2008;37:300-305.

40. Glassman M, Hofmeister E, Weh JM, et al. Radiographic quantitative assessment of caudal proximal tibial angulation in 100 dogs with cranial cruciate ligament rupture. Vet Surg 2011;40:830-838.

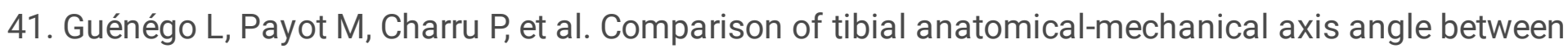
predisposed dogs and dogs at low risk for cranial cruciate ligament rupture. Vet $J$ 2017;225:35-41.

42. Abel SB, Hammer DL, Shott S. Use of the proximal portion of the tibia for measurement of the tibial plateau angle in dogs. Am J Vet Res 2003;64:1117-1123.

43. Warzee CC, Dejardin LM, Arnoczky SP, et al. Effect of tibial plateau leveling on cranial and caudal tibial thrusts in canine cranial cruciate-deficient stifles: an in vitro experimental study. Vet Surg 2001;30:278-286.

44. Jerram RM, Walker AM. Cranial cruciate ligament injury in the dog: pathophysiology, diagnosis and treatment. N Z Vet J 2003;51:149-158.

45. Henderson RA, Milton JL. The tibial compression mechanism: a diagnostic aid in stifle injuries. $J$ Am Anim Hosp Assoc 1978;14:474-479. 
46. Fuller MC, Hayashi K, Bruecker KA, et al. Evaluation of the radiographic infrapatellar fat pad sign of the contralateral stifle joint as a risk factor for subsequent contralateral cranial cruciate ligament rupture in dogs with unilateral rupture: 96 cases (2006-2007). J Am Vet Med Assoc 2014;244:328338.

47. Chuang C, Ramaker MA, Kaur S, et al. Radiographic risk factors for contralateral rupture in dogs with unilateral cranial cruciate ligament rupture. PLoS One 2014;9.

48. Rayward RM, Thomson DG, Davies JV, et al. Progression of osteoarthritis following TPLO surgery: a prospective radiographic study of 40 dogs. J Small Anim Pract 2004;45:92-97.

49. Hayes GM, Granger N, Langley-Hobbs SJ, et al. Abnormal reflex activation of hamstring muscles in dogs with cranial cruciate ligament rupture. Vet J 2013;196:345-350.

50. Fujita $\mathrm{Y}, \mathrm{Hara} \mathrm{Y}$, Ochi $\mathrm{H}$, et al. The possible role of the tibial plateau angle for the severity of osteoarthritis in dogs with cranial cruciate ligament rupture. J Vet Med Sci 2006;68:675-679.

51. Ichinohe T, Kanno N, Harada Y, et al. Degenerative changes of the cranial cruciate ligament harvested from dogs with cranial cruciate ligament rupture. J Vet Med Sci 2015;77:761-770.

52. Chuang C, Ramaker MA, Kaur S, et al. Radiographic risk factors for contralateral rupture in dogs with unilateral cranial cruciate ligament rupture. PLoS One 2014;9:1-10.

53. Slocum B, Slocum TD. Tibial plateau leveling osteotomy for repair of cranial cruciate ligament rupture in the canine. Vet Clin North Am Small Anim Pract 1993;23:777-795.

54. Slocum B, Slocum TD. Tibial plateau leveling osteotomy for cranial cruciate ligament rupture. Curr. Tech. Small Anim. Surgery, 4th ed 1209- 1215 (1998).

55. Reif U, Hulse DA, Hauptman JG. Effect of tibial plateau leveling on stability of the canine cranial cruciate-deficient stifle joint: an in vitro study. Vet Surg 2002;31:147-154.

56. Warzee, CC, Dejardin LM, Arnoczky SP, et al. Effect of tibial plateau leveling on cranial and caudal tibial thrusts in canine cranial cruciate-deficient stifles: an in vitro experimental study. Vet Surg 2001;30:278-286.

57. Read RA, Robins GM. Deformity of the proximal tibia in dogs. Vet Rec 1982;111:295-298.

58. Dejardin L. Tibial plateau levelling osteotomy. Textbook of Small Animal Surgery. Slatter D, editor. 3rd ed. Philadelphia: Saunders; 2003.

59. Jeffcott LB. The stifle joint. Equine Vet J 2010;18:15-22.

60. PB, V. Stifle joint. Textbook of Small Animal Surgery. Slatter D, editor. 3rd ed. Philadelphia: Saunders; 2003.

61. Ichinohe, T, Kanno N, Harada Y, et al. Histological and immunohistological analysis of degenerative changes in the cranial cruciate ligament in a canine model of excessive tibial plateau angle. Vet Comp Orthop Traumatol 2015;28:240-249.

62. Fujita $\mathrm{Y}$ Hara $\mathrm{Y}$, Ochi H, et al. The possible role of the tibial plateau angle for the severity of osteoarthritis in dogs with cranial cruciate ligament rupture. J Vet Med Sci 2006;68:675-679. 
63. Dejour $\mathrm{H}$, Bonnin M. Tibial translation after anterior cruciate ligament rupture. Two radiological tests compared. J Bone Joint Surg Br 1994;76:745-749.

64. Fritschy D, Panoussopoulos A, Wallensten R, et al. Can we predict the outcome of a partial rupture of the anterior cruciate ligament? A prospective study of 43 cases. Knee 1997;5:2-5.

65. Johnson JM, Johnson AL. Cranial cruciate ligament rupture. Vet Clin North Am Small Anim Pract 1993;23:717-733.

66. Henderson RA, Milton JL. The tibial compression mechanism: a diagnostic aid in stifle injuries. J Am Anim Hosp Assoc 1978;14:474-479.

67. Scavelli TD, Schrader SC, Matthiesen DT, et al. Partial rupture of the cranial cruciate ligament of the stifle in dogs: 25 cases (1982-1988). J Am Vet Med Assoc 1990;196:1135-1138.

\section{Figures}

\section{Figure 1}
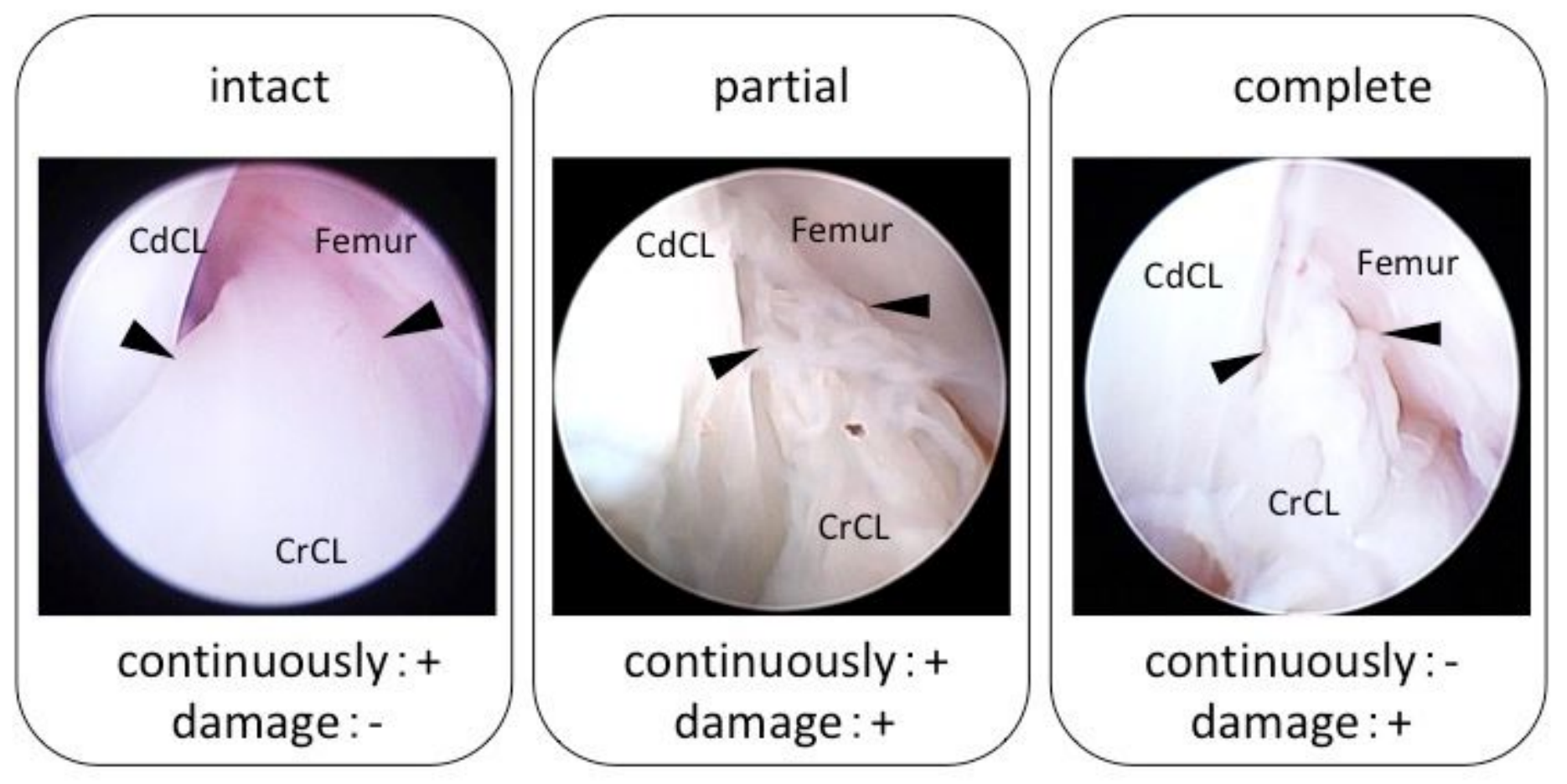

Figure 1 
Evaluation of the degree of damage to the cranial cruciate ligament by arthroscopy, i.e., intact (no damage, with continuity, partial tear (with damage and continuity, no ligament is completely ruptured, but none is intact), or complete tear (damage, no continuity). The majority of lesions were close to the femoral insertion site with no distal lesion.

\section{Figure 2}

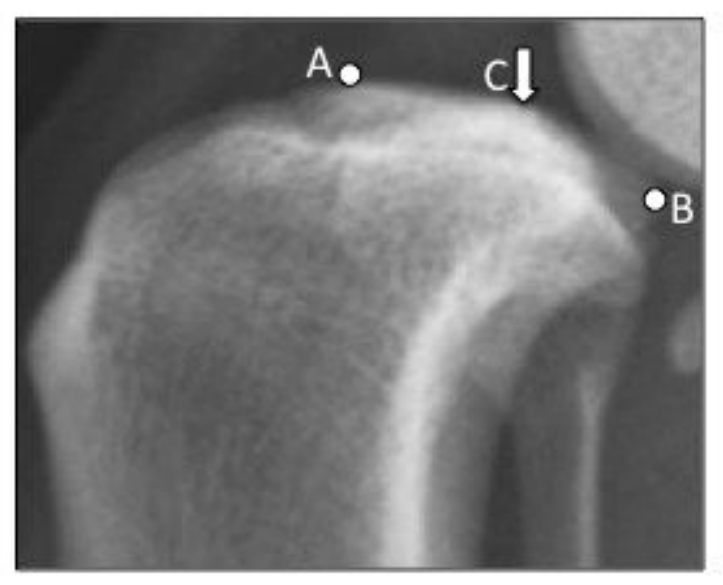

\section{Figure 2}

Proximal medial aspect of tibia. The cranial (dot A) and caudal (dot B) extents of the medial tibial condyle represent the landmarks for the proximal tibial joint orientation line in the sagittal plane. A point midway between the apices of the two tibial intercondylar eminences labeled with arrow (C) denotes the center of the stifle joint and the proximal reference mark for the sagittal mechanical axis. 


\section{Figure 3}

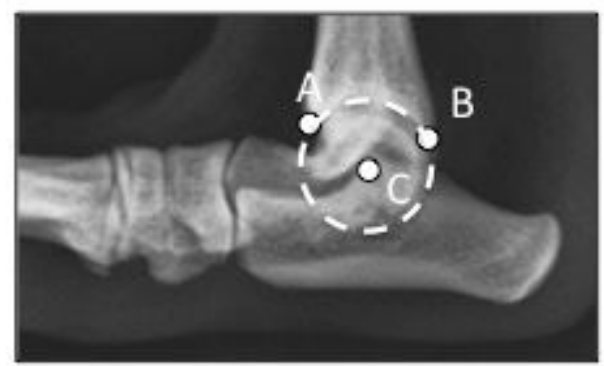

\section{Figure 3}

Distal medial aspect of tibia. The distal aspect of the distal intermediate ridge of the tibia cranially (dot A), and the caudodistal aspect of the cochlea tibia (dot B) represent the distal tibial joint orientation line in the sagittal plane. The center of the circle (C) created by the talus, which is represented by the broken circle, marks the distal reference mark for the mechanical axis in the sagittal plane. 


\section{Figure 4}

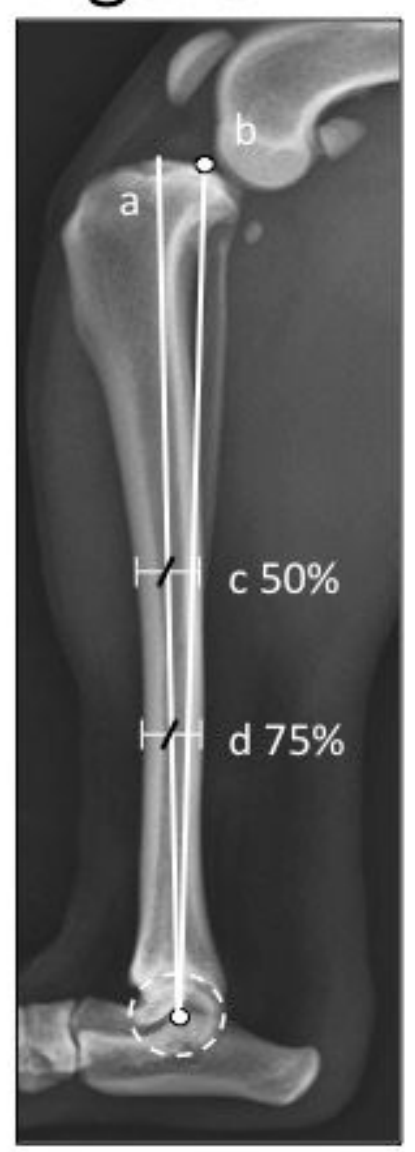

\section{Figure 4}

Entire tibia from the medial aspect, including the proximal and distal joint orientation lines and measurement of the anatomic and mechanical axes. The anatomic axis (a) was defined as the line formed by connecting the midpoint between the cranial and caudal cortex at $50 \%$ (c) and $75 \%$ (d) of the tibial shaft length. The mechanical axis (b) is a line extending from a point proximally dividing the medial and lateral intercondylar tubercles of the tibial plateau and a point equidistant to the cranial and caudal aspects of the trochlea of the talus. 


\section{Figure 5}

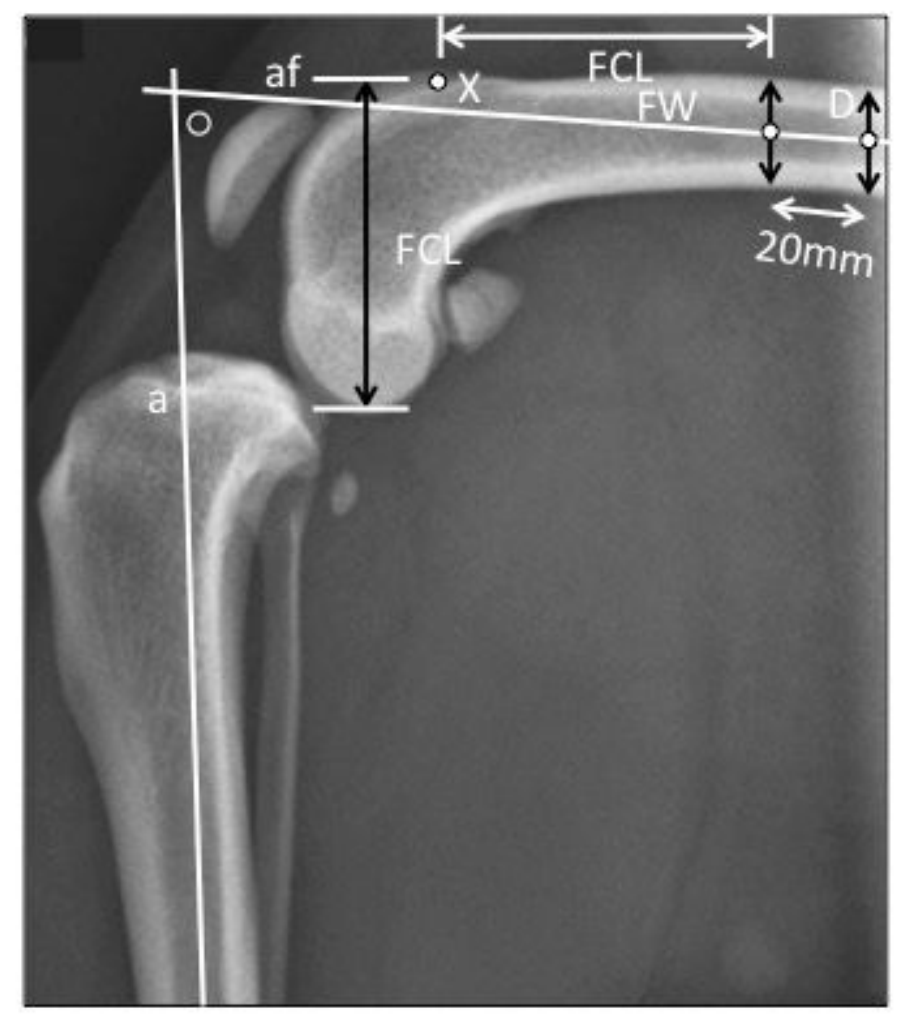

\section{Figure 5}

Measurement of flexion angle of the stifle joint. Measurement of stifle joint angle on a mediolateral radiograph view of a dog to illustrate measurement of the angles. The flexion angle (0) of the stifle joint was measured between the long axis of the femur (af) and the tibial anatomic axis (a). X, proximal extent of the femoral trochlea; a, the anatomical axis of tibia; FCL, femoral condylar length; FW, femoral width, which is measured at a distance equal to FCL from X; D, a segment parallel to $F W$ and drawn $20 \mathrm{~mm}$ proximal to FW. 


\section{Figure 6}

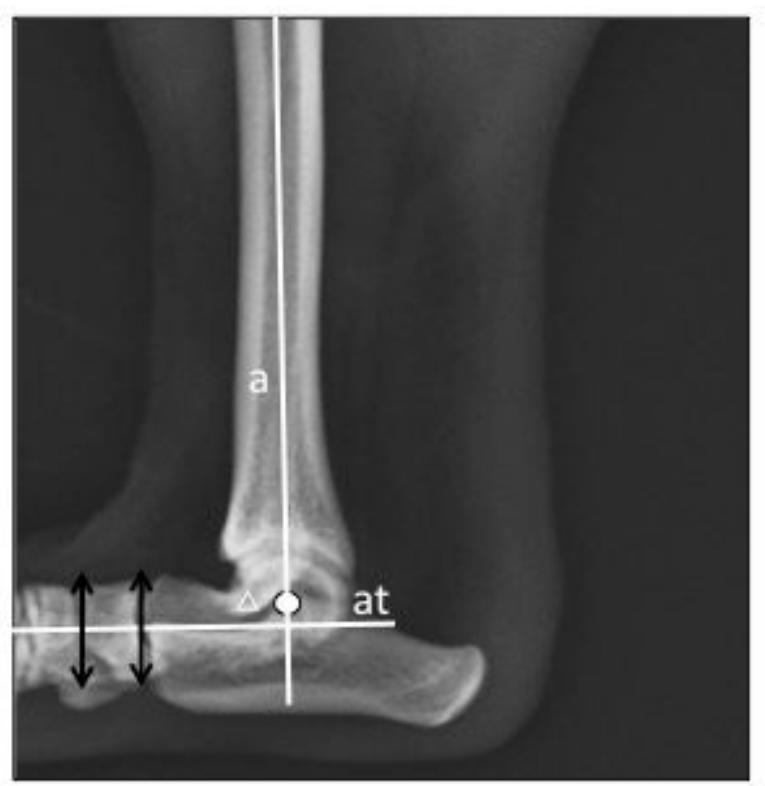

\section{Figure 6}

Measurement of flexion angle of the tarsal joint. Mediolateral radiographic view of a dog with a tarsal joint to illustrate measurement of the angle. Angle $\triangle$ was defined as the angle between the long axis of the tarsus (at) and tibial the anatomic axis (a). at, tarsal axis defined as the lines between the two shafts, midpoints at the intertarsal joint and the tarsal over the metacarpal joint 


\section{Figure 7}
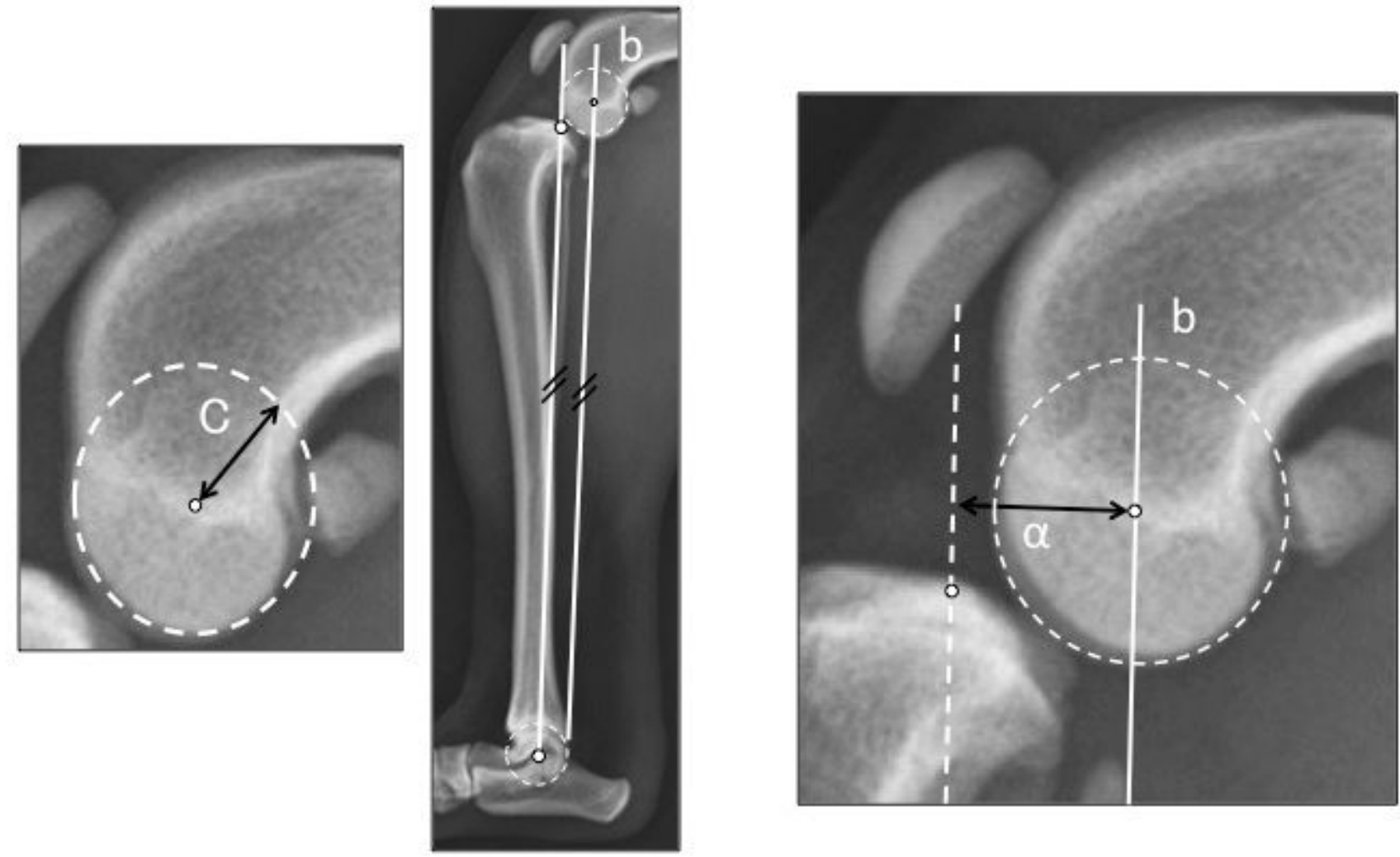

\section{Figure 7}

Measurement of the tibial cranial displacement index. A circle to fit the circumference of the femoral condyles (average of the circle for both the lateral and medial femoral condyles, more fit in the femoral condyles) was drawn, and then the center of the femoral condyle was determined and the radius of the femoral condyle was measured (C). A parallel line was drawn from the center of the femoral condyle to the tibial mechanical axis line. The distance (a) between these two points was measured. The ratio of a to $C$, known as the Tibial cranial Displacement Index (CTDI), was measured. 
Figure 8

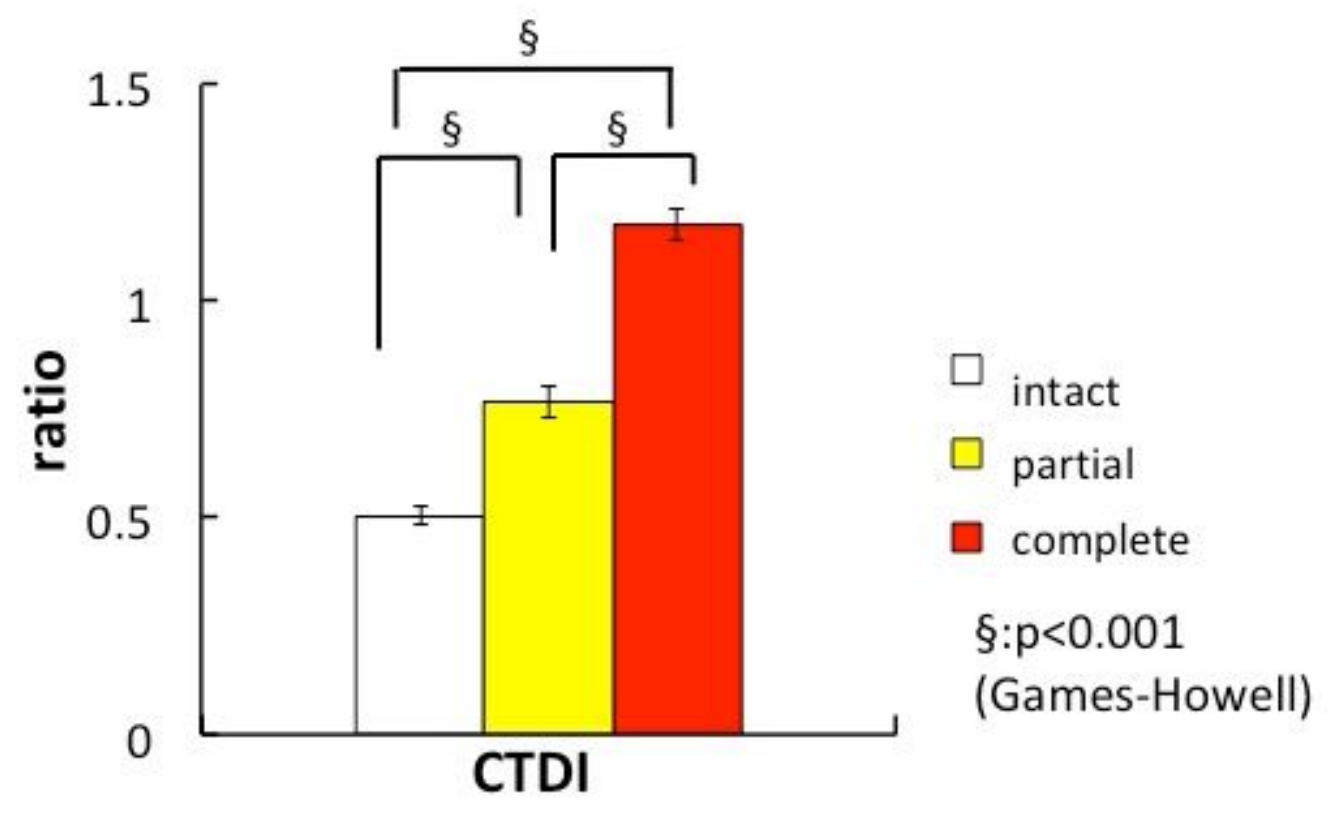

Figure 8

Outcome of measurement of the Tibial Cranial Displacement Index in the intact, partial rupture, and complete rupture groups. 
Figure 9

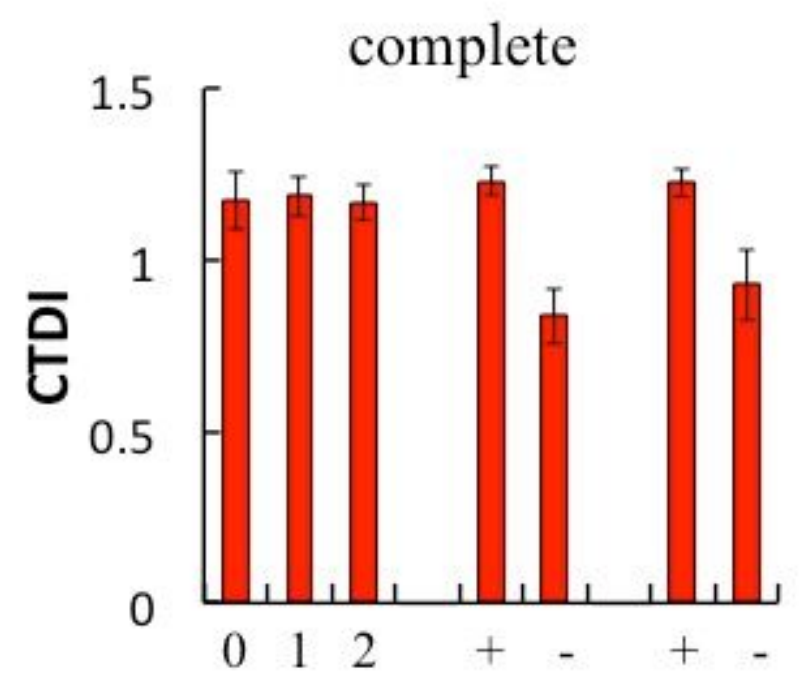

Fat pad sign CDT TCT

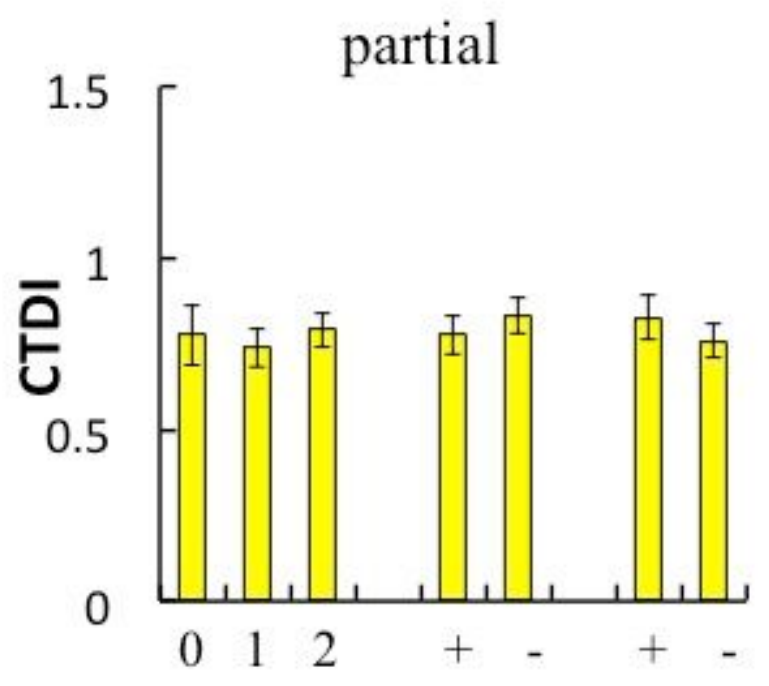

Fat pad sign CDT TCT

Figure 9

Comparison of the Tibial Cranial Displacement Index for each test. 


\section{Figure 10}

\section{normal \\ partial \\ complete}

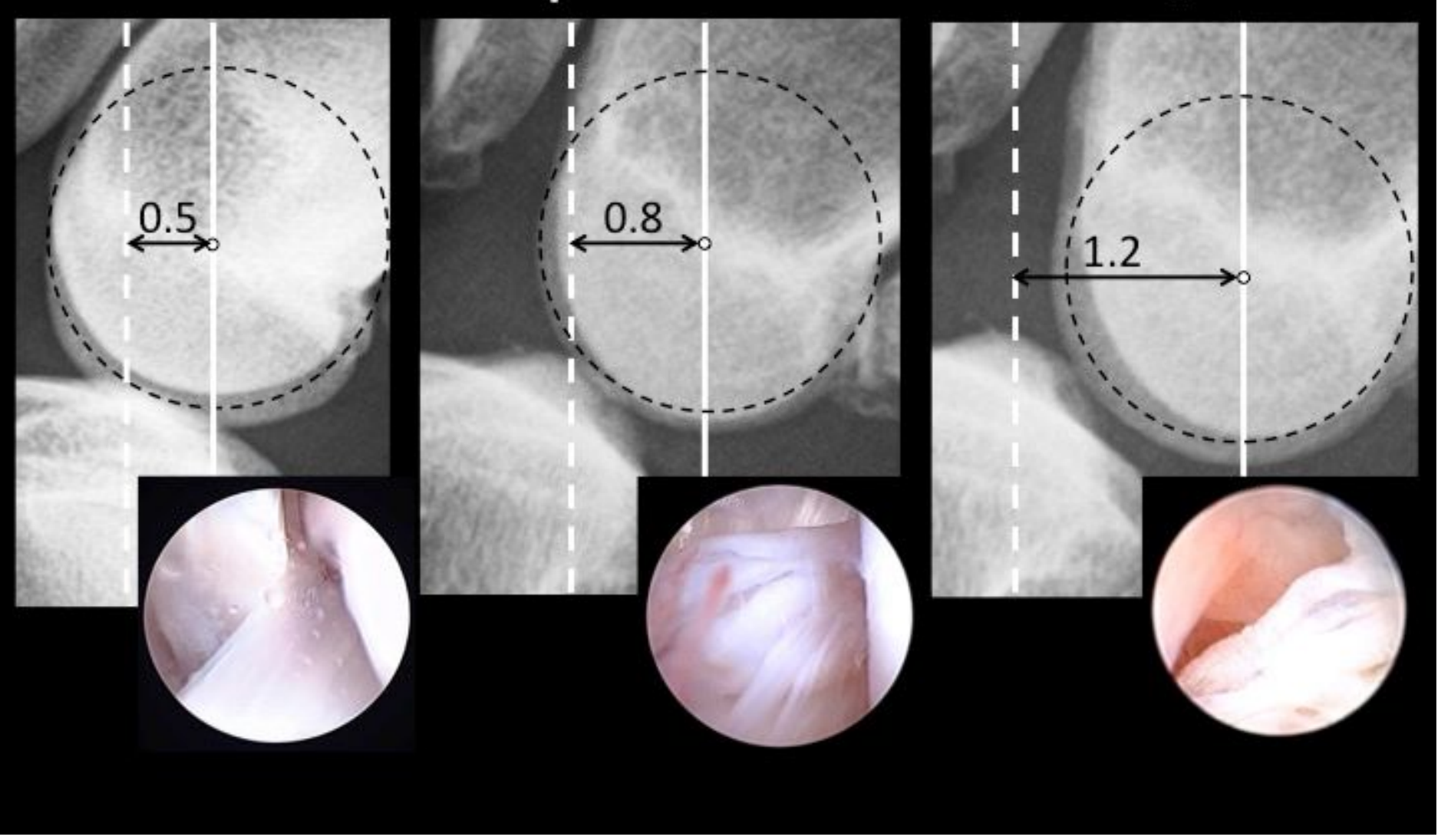

Figure 10

Comparison of Tibial Cranial Displacement Index score, radiographic results, and arthroscopic findings at the stifle joint between each of the three study groups. 